\title{
Welfarism in Economic Domains ${ }^{1}$
}

\author{
M. Ginés \\ Dept. Economia, Universitat Jaume I, I2071 Castellon, Spain \\ mgines $(a$ eco.uji.es \\ and \\ F. Marhuenda \\ Departamento de Economia, Universitat Carlos III, Madrid 126, 28903 Getafe, Spain \\ marhuend $a$ eco.uc 3 m.es

\begin{abstract}
In economies with public goods, and agents with quasi-linear preferences, we give a characterization of the welfare egalitarian correspondence in terms of three axioms: Pareto optimality, symmetry, and solidarity. This last property requires that an increase in the willingness to pay for the public goods of some of the agents should not decrease the welfare of any of them. Journal of Economic Literature
\end{abstract} \\ Classification Numbers: D63; H41
}

\section{INTRODUCTION}

The welfarist approach to Social Choice Theory proposes allocating resources in such a way that all the information not contained in the utility possibility set is ignored. This approach is exemplified in Bargaining Theory which studies utility allocation mechanisms defined on an abstract setting in which all the relevant information about the agents is summarized in the utility possibility set and a threat point. Characterizing those mechanisms by a minimal set of fairness properties is at the core of the theory.

${ }^{1}$ We thank L. Corchón, E. Dierker, J. Massó, F. Mertens, J. Naeve, W. Trockel, and an anonymous referee for their comments. We are especially indebted to J. Silvestre for discussing with us an earlier draft and making insightful suggestions. Financial support from the Instituto Valenciano de Investigaciones Económicas and from the Spanish Ministry of Education Project PB 97-0131 is gratefully acknowledged. 
This line of research has been questioned by J. Roemer [13-15]. J. Roemer's critique to Axiomatic Bargaining Theory is based on the observation that much of the relevant economic information is lost when the problem is presented as one of dividing up utility.

He provides some examples of genuinely different economic models giving rise to the same utility possibility set and the same threat point. Yet the intuition provided by some economic environments may not be valid for others. Thus, it is hard to justify the usage of mechanisms which depend only on the information contained in the utility possibility set. In addition, empirical results [21] suggest that the notions of fairness observed in people are based on more information than just that contained in the attainable utilities.

In [14], J. Roemer characterizes five classical solutions of Axiomatic Bargaining Theory. He argues that to reconstruct the standard axiomatic characterizations of Bargaining Theory, one has to consider commodity spaces of unbounded dimension. And it is no longer clear that the economic analogues of the axioms of Bargaining Theory still characterize a solution in more realistic and smaller domains. Thus, he views his work as "...demonstrating the lengths to which one must go to preserve the axiomatic characterization of the standard bargaining mechanisms on economic environments" [ 14, p. 32]. He concludes that classical Bargaining Theory is unacceptable as a positive model of the bargaining process as well as a normative model of resource allocation.

The present paper is an attempt to reconcile some of the principles contained in Bargaining Theory with the work of J. Roemer by showing that E. Kalai's characterization [4] of the egalitarian solution can be transplanted from classical Bargaining Theory into some economically meaningful environments.

We consider a set of agents endowed with preferences on vectors of public goods and a single private good (money), which can be represented by quasi-linear utility functions with constant marginal utility in the private good. There is a commonly owned technology to produce public goods bundles using the private good as an input. The agents differ in their valuations of the public goods and the issue is to design a production plan and a financing scheme for it.

We adopt the point of view of social choice theory in that we seek a solution determined by some equitable properties. We focus on the three key properties considered by Kalai in his characterization of the egalitarian solution, Pareto efficiency, symmetry and monotonicity. The axiom of Pareto optimality needs no modification in our context but, the other two have to be reinterpreted within the economic situation at hand.

The intuition behind the axiom of symmetry is that agents which cannot be distinguished with the information available in the model should be treated equally. In Bargaining theory this means that if the utility 
possibility set is symmetric, then all the agents should end up with the same utility level. However, here, as in J. Roemer's example, it will be the case that different economic situations with genuinely different agents correspond to the same utility possibility set. Thus, in the context of our modeling, it seems more appropriate to postulate the following axiom: whenever all the agents have the same preferences, they should all pay the same amount of private good (of course, the level of the public goods enjoyed by the agents is, by definition, the same for all of them).

The monotonicity axiom requires that enlarging the set of alternatives available to the agents should not hurt any of them. The well known intuition supporting this principle is that if the pie gets larger, then everybody should benefit (perhaps differently) from it. In the quasi-linear world a bigger pie corresponds to having a larger surplus to share. However, all the agents contribute to the surplus. And it is possible that it becomes larger, because of the greater contributions of some of the agents, even though some others reduce their participation in the common project. Is it fair then to demand axiomatically that all of them benefit? To make monotonicity more palatable we consider the following modification. If some agent raises his valuation of the public goods but the rest do not modify their valuations (so total surplus is now higher and nobody contributes less than before to it), then the payoff of every agent should not decrease.

This idea is not entirely new in the literature. It has been used before in [16-18] in a slightly different form. The reasoning therein is that whenever there is a change in the preferences of some agents, the ones whose preferences remain the same should be affected in the same direction. This property is usually referred to as "solidarity" and that is the name we have adopted here as well. This concept is also related with the notions of population solidarity [20] and skill solidarity [3].

The main result of our work is to show that on the set of economies with quasi-linear preferences, the three axioms we have just discussed determine the same rule as in classical Bargaining Theory. That is, the welfare egalitarian correspondence which splits the surplus equally among the agents. We restrict ourselves to standard economic domains with, for example, a fixed commodity space and make use of a reduced number of axioms. Thus, our findings provide (as in [13-15]) an alternative foundation of welfarism based on economic principles.

There is a rather extensive literature studying the egalitarian solution in addition to Kalai's characterization. R. B. Myerson [11] uses a condition on decomposability with respect to sequences of bargaining problems and enough invariance under ordinal utility transformations. W. Thomson [20] provides another characterization in terms of population monotonicity and other axioms. Moulin [8] considers social choice functions which share equally the surplus above a reference utility level. In a related work, 
H. Moulin and J. Roemer [9] propose three properties, in addition to efficiency, which reflect the public and private property rights of the agents.

Y. Sprumont [17] has axiomatized the welfare egalitarian solution by means of solidarity with respect to changes in the feasibility constraints and preferences. Whenever a change occurs in the feasibility correspondence and/or some of the agent's preferences, all the agents whose preferences have not changed are similarly affected. In contrast, the axiom of solidarity, as presented here, applies only to a restricted class of changes in the preferences of the agents. In addition, we define welfare egalitarianism in terms of the preferences of the agents themselves and not with respect to some abstract preordering on the space of preferences and indifference classes of allocations as in [17]. In other words, we insist that the modeling and axioms stem directly from the fundamentals of the economic scenario.

One may conclude that Bargaining Theory is a rather ambitious project with a scope which is unrealistically universal. After all, its proposals apply, in principle, to every conceivable conflict. Yet, some of its ideas and intuitions are recoverable if we are willing to work at a smaller scale and incorporate the relevant economic considerations into the model. Of course, the price one has to pay is a loss in the universality of the fairness properties, which might now depend on the economic context of interest.

\section{THE MODEL}

We consider an economy with one private good and several public goods. The consumption set of the private good is $\mathbb{R}$ and the space of public goods ${ }^{2}$ is $Y=\mathbb{R}_{+}^{m}=\left\{y \in \mathbb{R}^{m}: y \geqslant 0\right\}$. These are produced by means of a technology jointly owned by the agents. The production possibilities are described by a function $c: Y \rightarrow \mathbb{R}_{+}$measuring the cost of producing each bundle of public goods in terms of the single private good of the economy. Throughout this paper we will consider a fixed cost function $c$ satisfying the following.

Assumption 2.1. The mapping $c$ is lower semicontinuous ${ }^{3}$, non decreasing, satisfies $c(0)=0$ and

$$
\liminf _{\|y\| \rightarrow+\infty} \frac{c(y)}{\|y\|}=+\infty .
$$

\footnotetext{
${ }^{2}$ Given two vectors $x, z$ in some Euclidean space, $\mathbb{R}_{+}^{p}$, the notation $x \geqslant z$ (resp. $x \gg z$ ) means that $x_{i} \geqslant z_{i}$ (resp. $x_{i}>z_{i}$ ) for every $i=1, \ldots, p$. We write $x>z$ to indicate that $x \geqslant z$ and $x \neq z$. Finally, $x \neq z$ means that $x_{i} \leqslant z_{i}$ for some $i=1, \ldots, p$.

${ }^{3}$ A mapping $c: Y \rightarrow \mathbb{R}$ is lower semicontinuous if for each $z \in Y$ we have $f(z) \leqslant \lim \inf _{y \rightarrow z} f(y)$. A lower semicontinuous function is bounded below on every compact set and attains its minimum value.
} 
Assumption 2.1 allows for technologies with jumps, so initial fixed costs are not ruled out in the model. The limit condition precludes increasing returns to scale in the economy for very large bundles of public goods, but does not restrict the technology on a bounded set of goods.

We let $N=\{1,2 \ldots, n\}$ denote the set of agents with consumption set $X_{i}=\mathbb{R}$, for $i \in N$. We let $X=X_{1} \times \cdots \times X_{n}$. An allocation $(z ; t)=$ $\left(z ; t_{1}, \ldots, t_{n}\right) \in Y \times X$ is feasible whenever $c(z) \leqslant \sum_{i=1}^{n} t_{i}$ and $t_{i} \leqslant \omega_{i}$ for all $i \in N$. Here, $\omega_{i}$ is the initial endowment of private good of agent $i \in N$. The preference relation of agent $i=1, \ldots, n$ is represented by a quasi-linear utility function ${ }^{4} u_{i}(y ; t)=\pi_{i}(y)+\omega_{i}-t_{i}$, with $(y ; t)=\left(y ; t_{1}, \ldots, t_{n}\right) \in Y \times X$ and $\pi_{i}: Y \rightarrow \mathbb{R}$. Hence, $u_{i}(y ; t)$ is the utility obtained by agent $i \in N$ when the bundle $y \in Y$ of public goods is implemented and he has to contribute the amount $t_{i}$ towards, its financing. For convenience, we write $u_{i}(y ; t)$ as depending on $t=\left(t_{1}, \ldots, t_{n}\right) \in X$ even though agent $i \in N$ is interested only in the consumption of the public goods and his private good so, for $\pi_{1}, \ldots, \pi_{n}$ and $\omega_{1}, \ldots, \omega_{n}$ fixed, $u_{i}(y ; t)$ depends only on $y \in Y$ and $t_{i} \in X_{i}$. The following assumption is made on the preferences of the agents.

Assumption 2.2. For each $i=1, \ldots, n, \pi_{i}: Y \rightarrow \mathbb{R}_{+}$is a continuous, nondecreasing function satisfying $\pi_{i}(0)=0$ and

$$
\limsup _{\|y\| \rightarrow \infty} \frac{\pi_{i}(y)}{\|y\|}<\infty .
$$

There are several interpretations for the mappings $\pi_{1}, \ldots, \pi_{n}$ and we do not adhere necessarily to any of them. On the one hand, the amount $\pi_{i}(y)$ represents the valuation that agent $i \in N$ has of the public goods $y$. One can also think of it as representing his private technology to exploit those public goods or the benefit (in terms of the private good) he would obtain if he could enjoy those public goods for free. If the status quo is no consumption of any of the public goods, then $\pi_{i}(y)$ is also the maximum amount of his private good that he is willing to pay for the consumption of the bundle $y$.

On the other hand, $\pi_{i}(y)+\omega_{i}-t_{i}$ is the net benefit agent $i \in N$ obtains when he has to contribute $t_{i}$ units of his private good in order to enjoy the bundle $y$ of public goods. Thus, $\pi_{i}(y)+\omega_{i}-t_{i}$ is also the net contribution that agent $i \in N$ makes towards the total surplus, $\sum_{i=1}^{n}\left(\pi_{i}(y)+\omega_{i}\right)-c(y)$, that the society obtains from the consumption of the bundle $y \in Y$ of public goods.

We refer to a vector of utility functions $\pi=\left(\pi_{1}, \ldots, \pi_{n}\right)$ as a profile of utilities and we use the notation $\omega=\left(\omega_{1}, \ldots, \omega_{n}\right)$ and $u(y ; t)=u^{(\pi, \omega)}(y ; t)=$ $\left(u_{1}(y ; t), \ldots, u_{n}(y ; t)\right)=\left(\pi_{1}(y)+\omega_{1}-t_{1}, \ldots, \pi_{n}(y)+\omega_{n}-t_{n}\right)$. The vector of

\footnotetext{
${ }^{4}$ Sometimes, we will write $u_{i}^{\pi}$ or $u_{i}^{\left(\pi, \omega_{i}\right)}$ to emphasize the dependence of $u$ on $\pi$ and/or $\omega$.
} 
utilities resulting from $\pi=\left(\pi_{1}, \ldots, \pi_{n}\right)$ when $\pi_{i}$ is replaced by the new utility function $v_{i}$ is denoted by $\left(\pi_{-i}, v_{i}\right)=\left(\pi_{1}, \ldots, \pi_{i-1}, v_{i}, \pi_{i+1}, \ldots, \pi_{n}\right)$ (and likewise, for endowments $\omega$ ). Given two utility profiles $\pi$ and $v$ defined on $Y$ we say that $\pi \geqslant v$ whenever $\pi(y) \geqslant v(y)$ for every $y \in Y$.

Definition 2.3. Given $\pi$ and $\omega$, a feasible allocation $(y ; t) \in Y \times X$ is Pareto optimal if there is no other feasible allocation $(z ; r) \in Y \times X$ such that $u(y ; t)<u(z ; r)$. The set of those Pareto optimal allocations $(y ; t) \in Y \times X$ satisfying $\pi_{1}(y)-t_{1}=\cdots=\pi_{n}(y)-t_{n}$ is the set of all welfare egalitarian allocations.

Note that the welfare egalitarian allocations equate the surplus obtained from the public goods ignoring the initial differences in endowments. Thus, we are considering $\omega$ to be the disagreement point or status quo: if the public project is not developed, every agent keeps his initial endowment of the private good and this is the minimum welfare level guaranteed by the welfare egalitarian correspondence. The solution proposed above suggests to equate the welfare gains of the agents above the disagreement point. An alternative definition might be to require that $\pi_{1}(y)+\omega_{1}-t_{1}=\cdots=$ $\pi_{n}(y)+\omega_{n}-t_{n}$. We will discuss other differences between these two possibilities at the end of Section 3.

The welfare egalitarian correspondence is essentially unique since the agents are indifferent among the various allocations in it. However, as the next example shows, it might be empty.

Example 2.4. Let us take the technology $c(y)=y^{2}$ and the utility functions

$$
\pi_{1}(y)=\left\{\begin{array}{ll}
y, & \text { if } 0 \leqslant y \leqslant 1 \\
1, & \text { if } y \geqslant 1
\end{array} \quad \pi_{2}(y)=\left\{\begin{array}{lll}
10 y & \text { if } \quad 0 \leqslant y \leqslant 1 \\
10 & \text { if } \quad y \geqslant 1
\end{array}\right.\right.
$$

Assume first, that the vector of initial endowments $\omega=\left(\omega_{1}, \omega_{2}\right)$ satisfies $1 / 4 \leqslant \omega_{1}+\omega_{2} \leqslant 1$. Then, one checks easily that the set of Pareto optimal allocations is given by $\left\{\left(y ; t_{1}, t_{2}\right): 1 / 2 \leqslant y, c(y) \leqslant \omega_{1}+\omega_{2}, t_{1}+t_{2}=c(y)\right.$, $\left.t_{2}=\omega_{2}\right\}=\left\{\left(y ; y^{2}-\omega_{2}, \omega_{2}\right): 1 / 2 \leqslant y \leqslant \sqrt{\omega_{1}+\omega_{2}}\right\}$. Hence, the set $U_{1}=$ $\left\{\left(\pi_{1}(y)-t_{1}, \pi_{2}(y)-t_{2}\right):\left(y ; t_{1}, t_{2}\right)\right.$ is Pareto optimal $\}=\left\{\left(y-y^{2}+\omega_{2}\right.\right.$, $\left.10 y-\omega_{2}\right): 1 / 2 \leqslant y \leqslant \sqrt{\left.\omega_{1}+\omega_{2}\right\}}$ does not contain any points of the form $\pi_{1}(y)-t_{1}=\pi_{2}(y)-t_{2}$, i.e., there are no welfare egalitarian allocations in this economy.

On the other hand, if $\omega_{1}+\omega_{2} \geqslant 1$, then the set $P=\left\{\left(y ; t_{1}, t_{2}\right): t_{1}+t_{2}=\right.$ $\left.1, t_{i} \leqslant \omega_{i}, i=1,2\right\}$ is also a subset of the Pareto optimal allocations. Thus, the set $U_{2}=\left\{\left(\pi_{1}(1)-t_{1}, \pi_{2}(1)-t_{2}\right):\left(y ; t_{1}, t_{2}\right) \in P\right\}=\left\{\left(1-t_{1}, 9+t_{1}\right): 1-\right.$ $\left.\omega_{2} \leqslant t_{1} \leqslant \omega_{1}\right\}$ is also a subset of the set of utilities attained via Pareto 
optimal allocations. And we see that as long as $\omega_{2} \geqslant 5$ there are $\left(y ; t_{1}, t_{2}\right) \in P$ for which $\pi_{1}(y)-t_{1}=\pi_{2}(y)-t_{2}$.

The above example illustrates two issues which arise from the introduction of initial endowments. The first one is rather technical: the Pareto optimal allocations do not necessarily correspond to different distributions of the cost of the bundle(s) optimizing the surplus $\sum_{i=1}^{n} \pi_{i}(y)-c(y)$ of the economy. This statement holds only for Pareto optimal allocations $(y ; t)$ for which $t \gg \omega$ (see [1]).

More substantially for our purposes, $\mathrm{t}$ shows that whenever the total resources $\omega_{1}+\cdots+\omega_{n}$ are scarce, it can be the case that the Pareto frontier does not contain any welfare egalitarian allocations. Since, these allocations are the focus of this work, we introduce next a new hypothesis which guarantees there existence.

Assumption 2.5. For each $y$ such that $\sum_{i=1}^{n} \pi_{i}(y)-c(y) \geqslant 0$, the inequalities ${ }^{5} \omega_{k}>\pi_{k}(y)$ for all $k \in N$ and $\sum_{i=1}^{n} \omega_{i}>c(y)$ hold.

By Assumption 2.5 there are enough private resources to carry out the optimal plan of public goods and to make payment transfers of the private good among the agents. Example 2.4 shows that the welfare egalitarian correspondence might be empty unless something like Assumption 2.5 holds. Nevertheless, we can dispose of Assumption 2.5 provided we are willing to allow negative consumption of the private good. ${ }^{6} \mathrm{We}$ will not do so, since in the present framework of interpersonal comparison of utilities, it seems reasonable to take into account the effects that the initial distribution of endowments might have on the welfare attained by the agents.

For a fixed technology $c$, for which Assumption 2.1 holds, an economy is defined to be a pair $(\pi, \omega)$ consisting of a vector of utility profiles $\pi=$ $\left(\pi_{1}, \ldots, \pi_{n}\right)$ verifying Assumption 2.2 and a vector of initial endowments $\omega=\left(\omega_{1}, \ldots, \omega_{n}\right)$ satisfying Assumption 2.5. We let $E$ denote the set of such economies. A mechanism is a function $R: E \rightarrow Y \times X$ which assigns to every economy $(\pi, \omega) \in E$ a feasible allocation $R(\pi, \omega)=(y(\pi, \omega) ; t(\pi, \omega))$. We denote by $\mathrm{P}(\pi, \omega)$ (resp. $W(\pi, \omega)$ ) the set of Pareto optimal (resp. welfare egalitarian) allocations.

Lemma 2.6. Let $(\pi, \omega) \in E$. Then, $W(\pi, \omega) \neq \varnothing$.

Proof. By Assumptions 2.1 and 2.2, there is a solution, say $\bar{y}$, to the maximization problem $\max \left\{\sum_{i=1}^{n} \pi_{i}(y)-c(y): y \in Y\right\}$. By Assumption 2.5, we have that $\bar{t}_{k}=\pi_{k}(\bar{y})-\frac{1}{n}\left(\sum_{i=1}^{n} \pi_{i}(\bar{y})-c(\bar{y})\right)<\omega_{k}$. Thus, the allocation

\footnotetext{
${ }^{5}$ By Assumptions 2.1 and 2.2, the inequality $\sum_{i=1}^{n} \pi_{i}(y)-c(y) \geqslant 0$ holds only on a bounded set of public goods.

${ }^{6}$ This is the standard assumption with quasi-linear preferences (see, for example, [7]).
} 
$(\bar{y} ; \bar{t})$ is feasible and satisfies $\pi_{i}(\bar{y})-\bar{t}_{i}=\pi_{j}(\bar{y})-\bar{t}_{j}$ for all $i, j \in N$. Since, it is Pareto optimal (see [1]) it is also welfare egalitarian.

The problem faced by the agents is to find "the optimal" bundle of public goods and a fair share of its cost. According to the normative approach an "acceptable" mechanism should satisfy certain equitable requirements. The principles which we propose here are described by the following three properties.

Axioms 2.7. For every $(\pi, \omega) \in E$,

(i) $R(\pi, \omega) \in \mathrm{P}(\pi, \omega)$.

(ii) If $\pi_{1}=\cdots=\pi_{n}$ and $\omega_{1}=\cdots=\omega_{n}$ then, $u_{1}(R(\pi, \omega))=\cdots=$ $u_{n}(R(\pi, \omega))$.

(iii) If $\pi \geqslant v$ and $\omega \geqslant \sigma$, then $u^{(\pi, \omega)}(R(\pi, \omega))-\omega \geqslant u^{(v, \sigma)}(R(v, \sigma))-\sigma$.

Properties (i) and (ii) reflect, respectively, the notions of Pareto efficiency and symmetry. They are standard in the literature, so we will make no further comment about them. The novelty here lies on Axiom 2.7 (iii). This is akin to the requirement of No Disposal of Utilities as stated in [8]. An equivalent statement is that if $\pi_{i_{0}} \geqslant v_{i_{0}}$ and $\omega_{i_{0}} \geqslant \sigma_{i_{0}}$, for some $i_{0} \in N$, then $u^{(\pi, \omega)}(R(\pi, \omega))-\omega \geqslant u^{(v, \sigma)}(R(v, \sigma))-\sigma$ for the vectors of utilities and endowments $\pi=\left(\pi_{-i_{0}}, v_{i_{0}}\right)$ and $\omega=\left(\omega_{-i_{0}}, \sigma_{i_{0}}\right)$. Thus, it reduces to comparisons involving only changes of preferences and endowments in one agent.

We note that Property 2.7(iii) focuses on increases in gains $u(R(\pi, \omega))$ $-\omega$, with respect to the status quo $\omega$, as $\pi$ and $\omega$ vary. We might also consider the possibility of studying changes in the total welfare $u(R(\pi, \omega))$ of the agents with respect to variations in $\pi$ and $\omega$. This is addressed at the end of the next section.

One possible interpretation of this axiom is that if, after reaching an agreement, one of the agents finds out that he can increase the benefit he obtains from the public goods, then he is entitled to a larger share of the surplus (since he contributes a greater amount to it) as long as this does not affect negatively the others. It is in this sense that Axiom 2.7 is called a solidarity axiom: An increase in the skill of one agent benefits the whole society, or at least does not hurt the other members. In particular, if one interprets the mappings $\pi_{1}, \ldots, \pi_{n}$ as the private technology used by the agents to exploit the public goods then, no agent will oppose technological advancement by others.

Thus, the axiom of solidarity is akin to the monotonicity property of Axiomatic Bargaining Theory. One may justify it on the basis that, since the technology to produce the public goods is jointly owned by all the agents, they are forced to cooperate in agreeing both on a single bundle of 
public goods and a financing plan for it. At the end of the next section we will come back to a further discussion of this issue.

Remark 2.8. To simplify the treatment, given a quasi-linear preference relation $\prec_{i}$, we are fixing the unique utility function representation $\pi_{i}(y)+\omega_{i}-t_{i}$ such that $\pi_{i}(0)=0$ and we work with this representation. Nevertheless, we point out that our solution concept $W$ and properties 2.7 can be given an ordinal meaning which is independent of the chosen representation.

For example, it is easy to show that property 2.7 (iii) is an ordinal axiom. To see this, consider for each agent $i \in N$, a (quasi-linear) preference relation $\prec_{i}$, defined on $Y \times \mathbb{R}$. Given $y \in Y$, define $T^{\prec_{i}}(y) \in \mathbb{R}$ to be the unique real number such that $(y, 0) \sim\left(0, T^{<i}(y)\right)$ (With the normalization above in place we have that $\left.T^{<i}(y)=\pi_{i}(y)\right)$. Then, Axiom 2.7(iii) can be stated as follows: Let $R(\prec, \omega)=(y(\prec, \omega) ; t(\prec, \omega))$ and $R\left(\prec^{\prime}, \sigma\right)=$ $\left(y\left(<^{\prime}, \sigma\right) ; t\left(\prec^{\prime}, \sigma\right)\right)$. If $\omega \geqslant \sigma$ and $T^{<i}(y) \geqslant T^{\alpha^{i}}(y)$ for all $y \in Y$ and $i \in N$, then $T^{\prec i}(y(\prec, \omega))-t_{i}(\prec, \omega) \geqslant T^{<i}\left(y\left(\prec^{\prime}, \sigma\right)\right)-t_{i}\left(\prec^{\prime}, \sigma\right)$ for all $i \in N$. Likewise, a feasible allocation $(\bar{y} ; \bar{t})$ is welfare egalitarian if and only if it is Pareto optimal and $T^{\alpha_{i}}(\bar{y})-\bar{t}_{i}=T^{<_{j}}(\bar{y})-\bar{t}_{j}$ for all $i, j \in N$.

As we have already noticed, even though $W(\pi, \omega)$ might contain several allocations, the agents are indifferent among them. Clearly any selection from $W$ satisfies Axioms 2.7. The content of the next result is that this is essentially the only way to obtain a mechanism satisfying those properties.

THEOREM 2.9. A mechanism $R$ satisfies Axioms 2.7 if and only if $R(\pi, \omega) \in W(\pi, \omega)$ for every $(\pi, \omega) \in E$.

The result above admits also a negative interpretation. One may ask whether it is possible that increasing the skill that some agents have to profit from the public goods benefits only (or perhaps mostly) those agents responsible for the larger surplus, without hurting the others. Theorem 2.9 shows that this is incompatible with Pareto efficiency and symmetry.

We address now the proof of Theorem 2.9. We will show that every mechanism satisfying Axiom 2.7 has to be a selection of the welfare egalitarian correspondence.

Proof of Theorem 2.9. Let $R$ be a mechanism satisfying Axiom 2.7 and let $R(\pi, \omega)=(\bar{y} ; \bar{r}) \in Y \times X$. The proof proceeds in three steps.

Step 1. Choose another economy with the same endowment $\omega$ and a profile of utilities $v(y)$ such that $v \leqslant \pi$ and $v_{i}(z)=\pi_{i}(\bar{y})$ is constant for every $z \geqslant \bar{y}$. Then, $u^{\pi}(\bar{y} ; \bar{r})=u^{v}(\bar{y} ; \bar{r})$ and by Axiom 2.7(iii) $u^{v}(R(v, \omega)) \leqslant$ $u^{\pi}(\bar{y} ; \bar{r})=u^{v}(\bar{y} ; \vec{r})$. Since, $R(v, \omega) \in \mathbf{P}(v, \omega)$ then $u^{\pi}(\bar{y} ; \bar{r})=u^{v}(R(v, \omega))$ and we may assume that $R(v, \omega)=(\bar{y} ; \bar{r})$ as well. 
Step 2. Choose $y^{*}$ large enough such that $y^{*} \gg \bar{y}$ and $c\left(y^{*}\right)>$ $n \max \left\{v_{1}(\bar{y}), \ldots, v_{n}(\bar{y})\right\}$. Let $\varepsilon>0$ be a real number such that $(1-\varepsilon) y^{*}$ $\gg \bar{y}$ and choose a utility profile $\xi(y)$ such that the following conditions hold.

(i) $\xi_{1}=\cdots=\xi_{n}$.

(ii) $\xi(z)=0$ for every $z \ngtr(1-\varepsilon) y^{*}$.

(iii) $\xi(z)=\xi\left(y^{*}\right)$ for every $z \geqslant y^{*}$.

(iv) For each $i \in N$, the solution to $\max \left\{\xi_{i}(z)-c(z): z \in Y\right\}$ is attained at the point $y^{*}$.

(v) $\xi(z)>v(z)$ for every $z \geqslant y^{*}$.

(vi) $\sum_{i=1}^{n} \xi_{i}\left(y^{*}\right)-c\left(y^{*}\right)=\sum_{i=1}^{n} v_{i}(\bar{y})-c(\bar{y})=\sum_{i=1}^{n} \pi_{i}(\bar{y})-c(\bar{y})$.

We indicate next (see Fig. 1) how it is possible to construct the functions $\xi_{1}, \ldots, \xi_{n}$. First, note that to obtain (iv) one only needs to make the functions $\xi_{1}=\cdots=\xi_{n}$ "steep" enough in the region between $(1-\varepsilon) y^{*}$ and $y^{*}$.

Choose now initial endowments $\sigma_{1}=\cdots=\sigma_{n}$ large enough so that $c\left(y^{*}\right) \leqslant \sum_{i=1}^{n} \sigma_{i}$. It follows from (iv) that the only Pareto optimal allocations are of the form $\left(y^{*} ; r^{*}\right)$ for some $r^{*} \in X$. Hence, $R(\xi, \sigma)=\left(y^{*} ; r^{*}\right)$ for some $r^{*} \in X$ such that $c\left(y^{*}\right)=\sum_{i \in N} r_{i}^{*}$. Now define $\xi_{1}\left(y^{*}\right)=\cdots=\xi_{n}\left(y^{*}\right)$ so that $\sum_{i=1}^{n} \xi_{i}\left(y^{*}\right)-c\left(y^{*}\right)=\sum_{i=1}^{n} v_{i}(\bar{y})-c(\bar{y}) \geqslant 0$. If necessary, increase $\sigma$ so that $\sigma_{i} \geqslant \xi_{i}\left(y^{*}\right)$. In addition, $\xi_{1}\left(y^{*}\right)+\cdots+\xi_{n}\left(y^{*}\right) \geqslant c\left(y^{*}\right)>$ $n \max \left\{v_{1}(\bar{y}), \ldots, v_{n}(\bar{y})\right\}$, so (v) also holds. Now (ii) and (iii) define the functions $\xi_{1}=\cdots=\xi_{n}$ on the whole space. It follows from (i) and Axiom 2.7(ii) that $r_{1}^{*}=\cdots=r_{n}^{*}$.

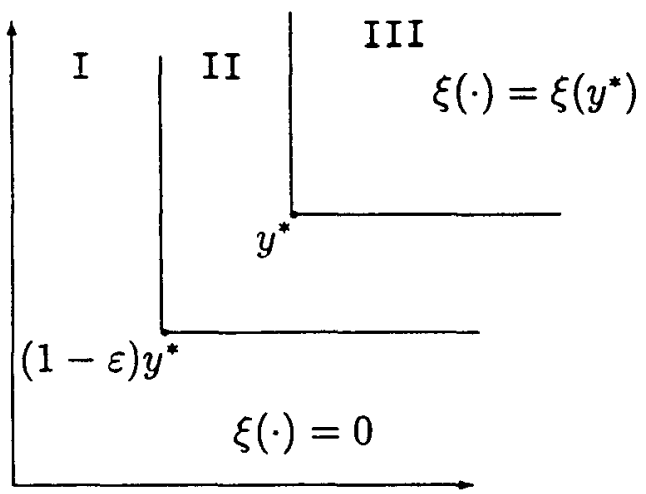

FIGURE 1 
Step 3. Take the vector of utilities $\beta_{i}(z)=\max \left\{v_{i}(z), \xi_{i}(z)\right\}, i=1, \ldots, n$ and the same endowments $\sigma$ as in step 2. We also choose $\varepsilon>0$ in there small enough so that, for each $i=1, \ldots, n$, the solution to the problem

$$
\left.\begin{array}{cc}
\max & \beta_{i}(y)-c(y) \\
\text { s.t. } & y \geqslant(1-\varepsilon) y^{*}
\end{array}\right\}
$$

is attained at the point $y^{*}$ (Note that, by $(\mathrm{v})$ in step $2, \beta(z)=\xi(z)$ if $z$ is close enough to $y^{*}$.)

Let $(\hat{y} ; \hat{r})=R(\beta, \sigma)$. The theorem will follow if we can prove that $\beta(\hat{y})-$ $\hat{r}=v(\bar{y})-\bar{r}=\xi\left(y^{*}\right)-r^{*}$. To see this, note first that by Axiom 2.7(iii) we have that $\beta(\hat{y})-\hat{r} \geqslant v(\bar{y})-\bar{r}$ and $\beta(\hat{y})-\hat{r} \geqslant \xi\left(y^{*}\right)-r^{*}$. We consider three cases.

(a) Assume first that $\hat{y} \geqslant y^{*}$. Then, by (v) we have that $\beta(\hat{y})=$ $\xi(\hat{y}) \geqslant v(\hat{y})$ and $\xi(\hat{y})-\hat{r}=\beta(\hat{y})-\hat{r} \geqslant \xi\left(y^{*}\right)-r^{*}$. Since $(\hat{y} ; \hat{r})=R(\beta, \sigma)$ is also feasible in the economy $(\xi, \sigma)$ and $\left(y^{*} ; r^{*}\right)=R(\xi, \sigma) \in \mathrm{P}(\xi, \sigma)$, then $\beta(\hat{y})-\hat{r}=\xi\left(y^{*}\right)-r^{*}$.

(b) Suppose now that $\hat{y} \ngtr(1-\varepsilon) y^{*}$. Then, from (ii) we see that $\beta(\hat{y})=v(\hat{y}) \geqslant \xi(\hat{y})$ and $v(\hat{y})-\hat{r}=\beta(\hat{y})-\hat{r} \geqslant v(\bar{y})-\bar{r}$. Hence, $\sum_{i=1}^{n} v_{i}(\hat{y})-$ $c(\hat{y}) \geqslant 0$ so, by Assumption 2.5, $c(\hat{y}) \leqslant \sum_{i=1}^{n} \omega_{i}$ and $\hat{y}$ is feasible in the economy $(v, \omega)$. But, if some inequality is strict, then $(\bar{y} ; \bar{r})$ would not be Pareto optimal in the economy $(v, \omega)$. Hence, $\beta(\hat{y})-\hat{r}=v(\bar{y})-\bar{r}=$ $\pi(\bar{y})-\bar{r}$.

(c) Otherwise, $\hat{y} \geqslant(1-\varepsilon) y^{*}$ and $\hat{y} \ngtr y^{*}$, so $\hat{y}$ is in region II of Fig. 1 . Then, by Eq. (2.1), we have $\xi_{i}\left(y^{*}\right)-c\left(y^{*}\right)=\beta_{i}\left(y^{*}\right)-c\left(y^{*}\right) \geqslant \beta_{i}(\hat{y})-c(\hat{y})$, for all $i \in N$. Since, $\beta(\hat{y})-\hat{r} \geqslant \xi\left(y^{*}\right)-r^{*}, c(\hat{y})=\sum_{i=1}^{n} \hat{r}_{i}$ and $c\left(y^{*}\right)=$ $\sum_{i=1}^{n} r_{i}^{*}$, it must be the case that $\beta(\hat{y})-\hat{r}=\xi\left(y^{*}\right)-r^{*}$.

In either of the three cases, the claim follows from $\sum_{i=1}^{n}\left(\xi_{i}\left(y^{*}\right)-r_{i}^{*}\right)=$ $\sum_{i=1}^{n}\left(v_{i}(\bar{y})-\bar{r}_{i}\right)$ and $\beta_{i}(\hat{y})=\max \left\{\xi_{i}(\hat{y}), v_{i}(\hat{y})\right\}$ for all $i=1, \ldots, n$. Thus, $\pi(\bar{y})-\bar{r}=\xi\left(y^{*}\right)-r^{*}$. But, by Axiom 2.7(iii), $\xi_{i}\left(y^{*}\right)-r_{i}^{*}=\xi_{j}\left(y^{*}\right)-r_{j}^{*}$ for all $i, j \in N$. Hence, we must also have $\pi_{i}(\bar{y})-\bar{r}_{i}=\pi_{j}(\bar{y})-\bar{r}_{j}$ for all $i, j \in N$, so $R(\pi, \omega) \in W(\pi, \omega)$ and the theorem follows.

\section{PRIVATE GOODS}

We argued in Section 2 that Axiom 2.3(iii) can be justified in a cooperative setting and it is interpreted as some type of solidarity among the agents. In this section we elaborate further on this issue and present a different economic context in which the characterization result of the 
previous section does not translate. As we will see, in an economic environment in which agents do not have any incentives to coordinate their decisions, the solidarity Axiom 2.3(iii) does no longer determine a unique solution. Thus, imposing this requirement has bite only whenever some degree of cooperation among the agents is necessary.

Formally (though not conceptually), the model we consider now is a slight modification of the one studied in the previous section. We abandon now the setting of public goods and let $Y_{i}=\mathbb{R}_{+}^{m}$ be the space of (produced) private goods consumed by agent $i \in N$. That is, we assume that the sets $X_{1}, \ldots, X_{n}$, represent, as in Section 2, the spaces of some private good which can be used, by means of a public technology $c$, to produce a bundle of goods $y \in \sum_{i=1}^{n} Y_{i}$. The key difference with the previous section is that the new vector $y$ is no longer a bundle of public goods, but it has to be divided $y=y^{1}+\cdots+y^{n} \in Y_{1}+\cdots+Y_{n}$ among the agents who consume them.

Thus, in contrast with Section 2, a feasible allocation consists now of a vector $\left(y^{1}, \ldots, y^{n} ; t\right) \in Y_{1} \times \cdots \times Y_{n} \times Y$ such that $t \leqslant \omega$ and $c\left(y^{1}+\cdots+y^{n}\right)$ $=t_{1}+\cdots+t_{n}$. The rest of the model and the assumptions made in the last section are translated readily into this new scenario. The question now is whether the equivalent of Theorem 2.9 holds in the setting of private goods.

To see that this is not the case consider a linear technology, so that $c(y+z)=c(y)+c(z)$. For each utility profile $u=\left(u_{1}, \ldots, u_{n}\right)$ and initial distribution of endowment $\omega$ we let, for each $i \in N,\left(y^{i}\left(u_{i}, \omega_{i}\right), t_{i}\left(u_{i}, \omega_{i}\right)\right) \in$ $Y_{i} \times X_{i}$ be the solution to agent $i$ 's maximization problem

$$
\left.\begin{array}{cc}
\max & u_{i}\left(y^{i}, t_{i}\right) \\
\text { s.t. } & c\left(y^{i}\right)=t_{i} \\
& t_{i} \leqslant \omega_{i}
\end{array}\right\} .
$$

Then, the mechanism $S: E \rightarrow Y_{1} \times \cdots \times Y_{n} \times X$ assigning the allocation $S(u, \omega)$ $=\left(y^{1}\left(u_{1}, \omega_{1}\right), \ldots, y^{n}\left(u_{n}, \omega_{n}\right) ; t_{1}\left(u_{1}, \omega_{1}\right), \ldots, t_{n}\left(u_{n}, \omega_{n}\right)\right)$ to every economy $(u, \omega)$, verifies properties 2.7 but is not welfare egalitarian.

The difference between public versus private goods is that in the first case agents are forced to come up with some common identical bundle, consumed by all of them. On the other hand, with private goods, the linear technology allows each of them to behave individualistically; in such a way that the different solutions proposed by each of the agents are compatible. This example shows that one has to be careful when postulating the principles of Bargaining Theory within economic environments. As pointed out by the work of Roemer [14], some of those axioms might be reasonable in some settings but completely unjustified for others. In particular, the characterization results might hold only for some, very concrete family of models but not for all them. In this sense, the price paid for getting around 
J.E. Roemer's critique and making the principles of Axiomatic Bargaining Theory applicable to economic scenarios is a loss in its universal character.

To finish we remark that our formulation for Axiom 2.7(iii) distributes the surplus above the initial endowments $\sum_{i=1}^{n} \pi_{i}(\bar{y})-c(\bar{y})$ equally among the agents and each agent $j \in N$ enjoys a utility equivalent to the consumption of no public good and the amount $\omega_{j}+\frac{1}{n}\left(\sum_{i=1}^{n} \pi_{i}(\bar{y})-c(\bar{y})\right)$ of the private goods. However the total surplus of the agents is in fact $S=$ $\sum_{i=1}^{n}\left(\pi_{i}(\bar{y})+\omega_{i}\right)-c(\bar{y})$. Another seemingly reasonable alternative might be to distribute total surplus $S$, including the initial endowment of private goods, among the agents so that all of them would end up with the same equivalent consumption of the private good. Let us call this solution $W^{*}$ so, $\left(y^{*} ; t\right) \in W^{*}(\pi, \omega)$ if it is Pareto optimal and $u_{i}\left(y^{*} ; t\right)=S / n$ for all agents $i \in N$.

It is easy to see that $W^{*}$ does not satisfy Property 2.7(iii). Nevertheless, it does comply with another very similar property which, at first sight, might be more natural than Property 2.7(iii). Namely,

$$
\text { if } \pi \geqslant v \quad \text { and } \quad \omega \geqslant \sigma, \quad \text { then } \quad u^{(\pi, \omega)}(R(\pi, \omega)) \geqslant u^{(v, \sigma)}(R(v, \sigma)) \text {. }
$$

Note that the original welfare egalitarian correspondence $W$ defined in Definition 2.3 also satisfies this alternative property. Hence, with this new axiom substituting Axiom 2.7(iii), there is no longer a unique solution. So, if we were to use the above property instead of Axiom 2.7(iii), we would need a fourth axiom to specify a solution. (One could, for example, specify what happens when there are no public goods.)

Let us argue that our proposal $W$ is more appropriate for the present set up than the other seemingly reasonable alternative $W^{*}$. We have just seen that, in the absence of public goods, equal distribution does not seem to have the same support in terms of the fairness principles that we have discussed here.

So, consider the extreme case in which public goods do not matter at all for the welfare of the society. That is, suppose that $\pi_{i}=0$ for all $i \in N$. For this particular example, our solution $W$ proposes the status quo allocation $(0 ; 0)$; i.e., no public good is implemented an each agent retains his initial endowment of private goods. On the other hand, $W^{*}$ would also dictate not to produce any public goods but it would still recommend to distribute the initial endowments equally among the agents. With no real incentives to cooperate, it does not seem so reasonable to expect that some agents would feel compelled to give away part of the their private endowment.

A similar argument shows also that, under the mechanism $W^{*}$, there will be situations in which some agents would be better off by keeping their initial endowment and not participating in the common project. Thus, $W^{*}$ fails also to provide some minimum individual rationality incentives. 


\section{REFERENCES}

1. T. C. Bergstrom and R. C. Cornes, Independence of allocative efficiency from distribution in the theory of public goods, Econometrica 51 (1983), 1753-1765.

2. Y. Chun, The solidarity axiom for quasi-linear social choice problems, Soc. Choice Welfare 3 (1986), 297-310.

3. M. Fleurbaey and F. Maniquet, Fair allocation with unequal production skills: The solidarity approach to compensation, mimeo University of Cergy-Pontoise, Cedex, December 1994

4. E. Kalai, Proportional solutions to bargaining situations: Interpersonal utility comparison, Econometrica 45 (1977), 1623-1630.

5. E. Kalai and D. Samet, Monotonic solutions to general cooperative games, Econometrica 53 (1985), 307-327.

6. H. Keiding and $\mathrm{H}$. Moulin, The solidarity axiom in parametric surplus-sharing problems, J. Math. Econ. 20 (1991), 249-270.

7. A. Mas-Colell, M. D. Whinston, and J. R. Green, "Microeconomic Theory," Oxford Univ. Press, London, 1995.

8. H. Moulin, Egalitarianism and utilitarianism in quasi-linear bargaining, Econometrica $\mathbf{5 3}$ (1985), 49-67.

9. H. Moulin and J. Roemer, Public ownership of the external world and private ownership of self, J. Polit. Econ. 97 (1989), 347-367.

10. H. Moulin and W. Thomson, Can everyone benefit from growth? Two difficulties, J. Math. Econ. 17 (1992), 339-345.

11. R. B. Myerson, Two-person bargaining problems and comparable utility, Econometrica 45 (1977), 1631-1637.

12. E. A. Pazner and D. Schmeidler, Egalitarian equivalent allocations: A new concept of economic equity, Quart. J. Econ. 92 (1978), 671-687

13. J. E. Roemer, Equality of resources implies equality of welfare, Quart. J. Econ. 101 (1986), $751-784$.

14. J. E. Roemer, Axiomatic bargaining theory in economic environments, J. Econ. Theory 45 (1988), 1-31.

15. J. E. Roemer, "Theories of Distributive Justice," Harvard Univ. Press, Cambridge, MA, 1996.

16. Y. Sprumont, Equal factor equivalence in economies with multiple public goods, mimeo Université de Montréal, Canada, July 1995.

17. Y. Sprumont, Axiomatizing ordinal welfare egalitarianism when preferences may vary, J. Econ. Theory 68 (1996), 77-110.

18. Y. Sprumont and L. Zhou, Pazner-Schmeidler rules in large societies, mimeo Université de Montréal, Canada, July 1995.

19. W. Thomson, Monotonic allocation rules in economies with public goods, mimeo University of Rochester, NJ, April 1989.

20. W. Thomson, Problems of fair division and the egalitarian solution, J. Econ. Theory 31 (1983), 211-226

21. M. Yaari and M. Bar-Hillel, On dividing justly, Soc. Choice Welfare 1 (1984), 1-24. 